\title{
PERCEPCIÓN SUBJETIVA DEL ACOSO SEXUAL EN LA FACULTAD DE CIENCIAS SOCIALES DE LA UNIVERSIDAD DEL SUR DE MÉXICO
}

Pedro Humberto Haddad Bernat ${ }^{1}$

\begin{abstract}
This document renders an account of the social perception of sexual harassment to which female student are subjected to in the Faculty of Social Sciences at the Southern University (Mexico). It shows the results of an internet poll which measures the perception that female students themselves have regarding who are the main perpetrators of sexual harassment, which behaviors are displayed by them, and how often. It has also been our interest to learn the perception that students have regarding the policies adopted by University's authorities in order to discourage sexual harassment.
\end{abstract}

Keywords: Gender Violence, Sexual Harassment, Sexism, University, Women.

\section{Introducción}

El presente trabajo consiste en la exposición de resultados de un estudio de percepción social del acoso sexual dirigido a estudiantes del sexo femenino en la Facultad de Ciencias Sociales (FCS, de aquí en adelante) de la Universidad del Sur de México (USM, de aquí en adelante).

Es importante recalcar desde el inicio que se ha elegido el nombre "Universidad del Sur" como sustituto del nombre real de la institución educativo cuya población de estudiantes fue objeto de investigación, por diversas razones que no es pertinente explicitar en este espacio. Sin embargo, es obvio que ello supone un gravamen metodológico en el que destaca, sobre todo, la lamentable imposibilidad para el autor de citar explícitamente las fuentes e informes oficiales (de rectoría, principalmente) de donde se obtienen los datos relativos a la

\footnotetext{
${ }^{1}$ Director del Centro de Especialización Judicial (Escuela Judicial) del Tribunal Superior de Justicia del Estado de Tabasco. Profesor de Instituto Prometeo para las Ciencias y las Artes. pedro.haddad@tsjtabasco.gob.mx
} 
población estudiantil total y a partir de los cuales se elabora la muestra.

Advertido lo anterior, este trabajo presentará los resultados de una encuesta electrónica realizada mediante un formulario de Google y distribuida por Whatsapp a 134 mujeres estudiantes de la FCS de la USM. Ello con el fin de conocer su percepción del acoso sexual, su incidencia, autoría frecuencia y demás datos sobre cómo lo han experimentado en el ambiente educativo en que se desenvuelven, de ser este el caso. Un objetivo ulterior que se persigue, aunque escapa los alcances de la presente exposición, es que estas estadísticas sirvan para poder diseñar propuestas de intervención para combatir y desincentivar el acoso sexual en el plantel educativo en cuestión.

\subsection{Justificación}

El acceso de las mujeres a una vida libre de violencia es una prioridad de toda sociedad que aspira a la más elemental decencia y uno de los grandes temas en la agenda política y social del siglo XXI. Es un derecho fundamental que deriva de la más simple comprensión y reconocimiento de las mujeres son seres humanos.
El acoso sexual es una forma violencia ejercida contra las mujeres que presupone una cultura hetero-patriarcal (Hoagland, 2000: 245) según la cual los cuerpos de las mujeres son tratados en la dinámica de las relaciones sociales, de manera consciente o inconsciente, como bienes para uso y disfrute de los hombres. La silenciosa y acrítica aceptación de dicha cultura (que se manifiesta o exhibe en una variedad de comportamientos) sitúa, obviamente, a las mujeres como clase en una condición social de singular vulnerabilidad.

El acoso sexual es una conducta (o conjunto de conductas) que manifiesta esa cultura de dominación del hombre sobre el cuerpo de la mujer y es especialmente lesiva cuando ocurre en el ambiente educativo, pues coloca a las mujeres en un estado de particular desventaja que obstaculiza su desarrollo intelectual y académico dentro de las expectativas de seguridad y confort que acompañan a la sana vida escolar.

El derecho de las mujeres de acceder a una vida libre de violencia tiene su fundamento en una serie de normas y principios del derecho internacional y del derecho nacional de diversos países. Puesto que el presente 
estudio se centra en el caso particular de una comunidad de estudiantes en el sureste de México, podemos señalar como algunos de los fundamentos jurídicos de este derecho a los que enunciaremos a continuación.

Así pues, en el contexto jurídico internacional, la Declaración Universal de los Derechos Humanos, reconocida como el instrumento principal en esa materia, dispone en su artículo primero que todas las personas nacemos libres e iguales en dignidad y derechos. Igualmente, el artículo segundo dispone que se deberá otorgar a las personas un trato digno sin distinción alguna de raza, color, sexo, idioma, religión, opinión política o de cualquier otra índole, origen nacional o social, posición económica, nacimiento o cualquier otra condición. También el artículo tercero reconoce que todo individuo tiene derecho a la vida, la libertad y la seguridad de su persona (Asamblea General Organización de las Naciones Unidad, 1948). Esta expresión es el corazón del principio de no discriminación, es decir, del trato igualitario a todas las personas sin importar sus diferencias.

Por su parte, la Convención Americana sobre Derechos Humanos,
188

recoge en su artículo primero la obligación de los Estados parte de respetar los derechos y libertades reconocidos en ella y a garantizar su libre y pleno ejercicio a toda persona que esté sujeta a su jurisdicción, sin discriminación alguna por motivos de raza, color, sexo, idioma, religión, opiniones políticas o de cualquier otra índole, origen nacional o social, posición económica, nacimiento o cualquier otra condición social (Asamblea General de la Organización de Estados Americanos, 1969).

Igualmente, en el escenario interamericano, se cuenta con la Convención Interamericana para prevenir, sancionar, y erradicar la violencia contra la mujer, conocida también como "Convención Belem do Pará" que es un instrumento en pro de garantizar que todas las mujeres puedan vivir una libre en la cual sea respetada su dignidad.

La relevancia de esta Convención estriba en que estipula conceptos como "violencia contra la mujer", que se define en su artículo primero como cualquier acción o conducta, basada en su género, que cause muerte, daño o sufrimiento físico, sexual 
o psicológico a la mujer, tanto en el ámbito público como en el privado.

El artículo segundo reconoce los tipos de violencia que pueden ser física, sexual y psicológica:

a. que tenga lugar dentro de la familia $o$ unidad doméstica $o$ en cualquier otra relación interpersonal, ya sea que el agresor comparta o haya compartido el mismo domicilio que la mujer, y que comprende, entre otros, violación, maltrato y abuso sexual;

b. que tenga lugar en la comunidad y sea perpetrada por cualquier persona y que comprende, entre otros, violación, abuso sexual, tortura, trata de personas, prostitución forzada, secuestro y acoso sexual en el lugar de trabajo, así como en instituciones educativas, establecimientos de salud o cualquier otro lugar, y

c. que sea perpetrada o tolerada por el Estado o sus agentes, dondequiera que ocurra.

Igualmente, en su artículo tercero reconoce que toda mujer tiene derecho a una vida libre de violencia, tanto en el ámbito público como en el privado (Asamblea General de la
Organización de Estados Americanos, 1994).

Ahora bien, en México se cuenta con una Ley General de Acceso a las Mujeres a una Vida Libre de Violencia (Estados Unidos Mexicanos, 2007), cuyo objeto es establecer la coordinación entre la Federación, las entidades federativas y los municipios para prevenir, sancionar y erradicar la violencia contra las mujeres, así como los principios y modalidades para garantizar su acceso a una vida libre de violencia que favorezca su desarrollo y bienestar conforme a los principios de igualdad y de no discriminación, así como para garantizar la democracia, el desarrollo integral y sustentable que fortalezca la soberanía y el régimen democrático establecidos en la Constitución Política de los Estados Unidos Mexicanos.

La misma norma, define en su numeral décimo la violencia laboral y docente explicando que se ejerce por las personas que tienen un vínculo laboral, docente o análogo con la víctima, independientemente de la relación jerárquica, consistente en un acto o una omisión en abuso de poder que daña la autoestima, salud, integridad, libertad y 
seguridad de la víctima, e impide su desarrollo y atenta contra la igualdad. Puede consistir en un solo evento dañino o en una serie de eventos cuya suma produce el daño. También incluye el acoso o el hostigamiento sexual.

Posteriormente, la Ley General de Acceso a las Mujeres a una Vida Libre de Violencia especifica en su numeral 12 que constituye violencia docente, aquellas conductas que dañen la autoestima de las alumnas con actos de discriminación por su sexo, edad, condición social, académica, limitaciones y/o características físicas, que les infligen maestras o maestros.

Atendiendo a todo este marco normativo, el propósito de fondo del estudio que aquí se presentará es el recabar los datos empíricos necesarios para establecer medidas de intervención en la realidad social encaminadas a cristalizar los fines e ideales establecidos por el marco jurídico. Recordemos que toda intervención social - para ser efectiva - debe de estar científicamente informada. Ello quiere decir que todo intento por cambiar un determinado espacio de la realidad social debe presuponer un debido estudio de dicho espacio mediante la aplicación de las
190

técnicas y métodos con las que la ciencia social nos provee. Así, en el tenor de lograr una ulterior intervención sobre el problema del acoso sexual sufrido por la comunidad de estudiantes objeto del presente estudio, he decidido mediante el este trabajo hacer una recolección de datos primarios de cara a hacer frente en una comunidad concreta en materia de un problema más general que aqueja a mujeres de todas las edades, condiciones y latitudes, si bien el diseño de dicha intervención no será objeto de estas líneas de exposición.

En aras de delimitar la muestra encuestada, el estudio se ciñe a un grupo de mujeres estudiantes de una universidad en la zona sureste de México, cerca de la frontera con Guatemala. Dicho centro educativo de nivel superior lo llamaremos para los efectos de este trabajo "Universidad del Sur de México" (USM) y el estudio comprende encuestas electrónicas realizadas sobre 134 estudiantes del sexo femenino. Las encuestas se realizaron utilizando la herramienta informática conocida como Google Forms (difundida utilizando la red social conocida como Whatsapp) y la población estudiada representa cerca del 
$10 \%$ de la población total de estudiantes del sexo femenino de la FCS de la mencionada casa de estudios.

\subsection{Objetivos}

Los objetivos de este trabajo son esencialmente tres:

a) Conocer si en la percepción social de la población estudiada hay un problema sistemático de acoso sexual sobre las estudiantes de la FCS de la Universidad del Sur y, en su caso, quienes son sus principales autores;

b) Conocer la percepción que la población estudiada tiene respecto de las conductas consideradas como formas de acoso sexual y la frecuencia con la que son perpetradas por los autores; y

c) Identificar el grado de conformidad o disconformidad que la población estudiada manifiesta respecto de la respuesta y tratamiento institucional de la situación.

\section{Metodología}

Esta investigación es de corte cuantitativo. Se realizó una recopilación de datos de fuente primaria, utilizándose como vehículo para la recogida de estos una encuesta electrónica conformada por
33 preguntas. El cuestionario fue desarrollado a través de un formulario de Google y fue distribuido por medio de un enlace compartido vía Whatsapp directamente entre miembros del grupo social de interés. Para ello, fue necesaria una aproximación personal previa con el fin de obtener el consentimiento de las estudiantes para colaborar con el estudio. En total, el enlace del cuestionario fue $\begin{array}{llll}\text { compartido exitosamente a } & 134\end{array}$ personas, mismas que constituyen la muestra que a continuación se describirá.

\subsection{Muestra}

La FCS, según el último informe anual de la Rectoría disponible en línea (2018), tiene una matrícula total de 2,803 estudiantes. De ellos, 2,668 son alumnos matriculados en alguna las tres licenciaturas que oferta la FCS: a) Derecho (2, 406 en modalidad presencial y 135 en modalidad $a$ distancia); b) Historia (81); y c) Sociología (181). De estos, un total de 1531 son estudiantes del sexo femenino. Para efectos de este estudio, se dejaron fuera 63 alumnas de la Licenciatura en Derecho en la modalidad a distancia quienes, por obvias razones, no participan de la vida cotidiana en el Campus. Así pues, se 
consideraron 1468 estudiantes como viables para la muestra. Hecha esta salvedad, la encuesta fue respondida por 134 mujeres estudiantes de licenciatura la FCS (lo que equivale a poco más de un 9\% de la población total), quienes compartieron su percepción respecto al acoso sexual que se vive dentro del campus universitario.

La tabla 1 revela cómo se conformó la muestra respecto a las edades de las encuestadas:

Tabla 1

\begin{tabular}{|l|l|}
\hline & Año de nacimiento \\
\hline 2 & 2001 \\
\hline 24 & 2000 \\
\hline 33 & 1999 \\
\hline 32 & 1998 \\
\hline 25 & 1997 \\
\hline 7 & 1996 \\
\hline 6 & 1995 \\
\hline 3 & 1994 \\
\hline 1 & 1992 \\
\hline 1 & 1987 \\
\hline
\end{tabular}


El $92.5 \%$ de las alumnas encuestadas cursaban al momento del estudio la Licenciatura en Derecho, el $6 \%$ la de Sociología y el $1.5 \%$, Historia. La población total de alumnas de la FCS se conforma de la manera señalada en la tabla 2. Cabe mencionarse que no se consideró parte de la población relevante para este trabajo a las alumnas de la Licenciatura en Derecho en modalidad $a$ distancia, dado que, por obvias razones, no son afectadas por el fenómeno que aquí nos ocupa de la misma manera que las que estudian en modalidad presencial. Dicho "corte" poblacional, por cierto, nos permite obtener una muestra al menos numéricamente más fiel a la población de interés, al consistir en prácticamente el $10 \%$ de la población estudiantil de sexo femenino (ver tabla 2).

Tabla 2

\begin{tabular}{|l|l|}
\hline Facultad & Alumnas \\
\hline Derecho & 1345 \\
\hline Historia & 28 \\
\hline Sociología & 95 \\
\hline Total & 1468 \\
\hline
\end{tabular}




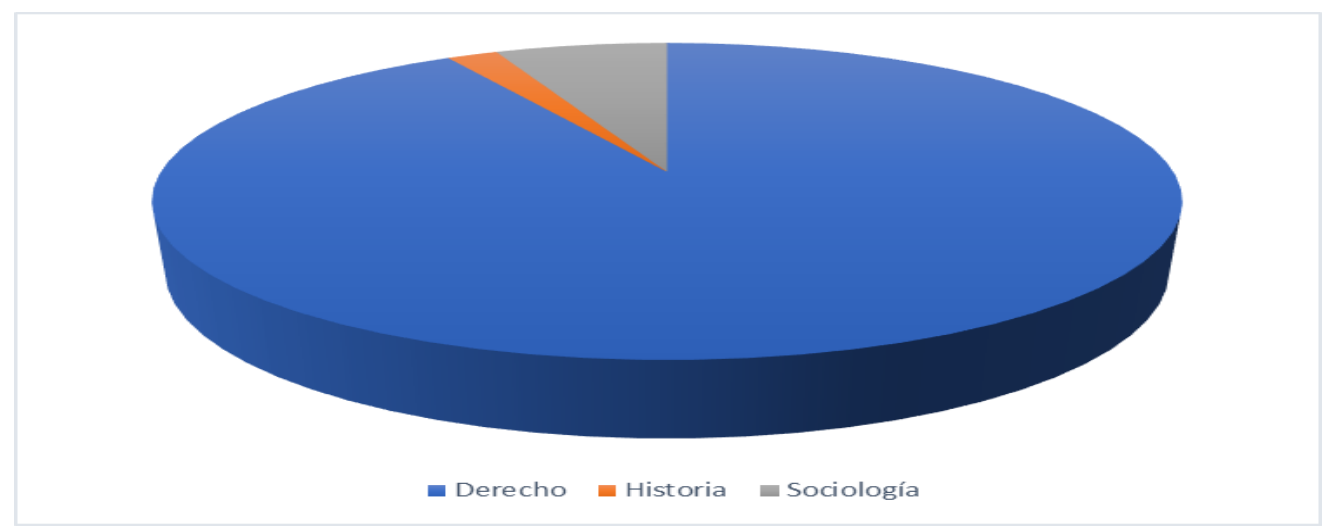

El $32,1 \%$ de las encuestadas dijo llevar entre 1 y 2 años estudiando, seguidas por un $25,4 \%$ con menos de un año. Así mismo, sólo el 3\% de las encuestadas afirmó tener alguna discapacidad. Y, por último, solo 1 de 134 estudiantes dijo ser hablante de alguna lengua indígena. Esa es la composición de la muestra que respondió el cuestionario acerca de la percepción social del acoso sexual dentro de la FCS de la USM.

\section{Resultados}

Fijadas las características de la muestra, a continuación, se presentarán los resultados de 27 preguntas restantes en el cuestionario y que constituyen el contenido nuclear del estudio.

En la segunda sección del cuestionario, a la que titulamos

"Comportamiento dentro de la clase", se observa en las preguntas 7 y 8 la percepción de las alumnas acerca de las bromas o comentarios que estereotipan o denigran a las mujeres, siendo el porcentaje que opina que las profesoras lo hacen frecuentemente un $16.4 \% \mathrm{y}$ muy frecuentemente $4.5 \%$, frente a un $33.6 \%$ que lo perciben como frecuente y $17.9 \%$ muy frecuente, por parte de los profesores. Un $43.3 \%$ de la muestra piensa que las profesoras no incurren nunca en estas conductas, mientras que sólo un $12.7 \%$ lo piensa así de los profesores.

Sobre las preguntas 9 y 10 igualmente fueron sobre comentarios o bromas que estereotipan a las personas, sólo que en esta ocasión a los hombres, y sobre ello la muestra manifestó percibir que el $61.9 \%$ de las profesoras lo hacen poco frecuentemente, mientras que un $8,2 \%$ indicó percibirlo como una 
conducta frecuente, al tiempo que por parte de los profesores a un $47.8 \%$ le parece que es poco frecuente por parte de los profesores, y a un $14.9 \%$ le parece una conducta que ocurre frecuentemente.

Subsecuentemente, se preguntó si dentro del aula las profesoras realizan bromas o comentarios de índole sexual que se consideraran inapropiadas. De lo obtenido, el 9.7 por ciento considero que se realizan de manera frecuente, mientras el $3.7 \%$ consideró que muy frecuentemente. Sin embargo, la respuesta dada a la misma pregunta, pero relativa a la frecuencia con que la conducta es realizada por los profesores, se obtuvo que el $35.1 \%$ de las alumnas la perciben como conducta frecuente y el $20.1 \%$ como muy frecuente.

En relación con los numerales 13 y del cuestionario, acerca de si en la clase se realizan preguntas incomodas acerca de la vida sexual o amorosa de las alumnas, ocurrió un fenómeno similar al de los numerales precedentes al señalar un $4.5 \%$ que perciben estas conductas frecuentemente y $3.7 \%$ muy frecuentemente por parte de las profesoras, delante de un $22.4 \%$ y $11.2 \%$ de los profesores, respectivamente. A continuación, se pueden consultar dos ilustraciones para facilitar la apreciación visual de estos datos:

\section{Ilustración 1}

13. Las profesoras hacen preguntas incómodas sobre la vida sexual o amorosa de las alumnas
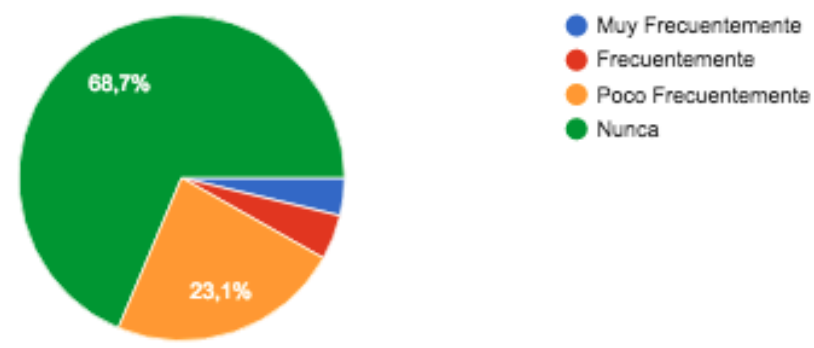


\section{Ilustración 2}

14. Los profesores hacen preguntas incómodas sobre la vida sexual o amorosa de las alumnas

134 respuestas
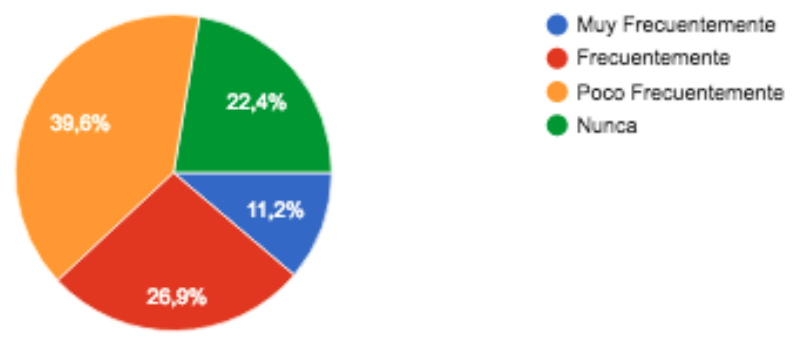

En tónica similar, se solicitó a la

3 a 9), se preguntó a las estudiantes población estudiada que manifestara su universitarias si han sido sujetos pasivos percepción acerca de piropos o de alguna de las diversas conductas comentarios no deseados respecto a la señaladas y que identificaran a quienes apariencia de las alumnas. De acuerdo perciben como los ejecutores de dichas con el $7.5 \%$ de las estudiantes, las conductas, estableciéndose como profesoras lo hacen frecuentemente y un opciones a: compañero estudiante, $3 \%$ manifiesta que muy frecuentemente compañera estudiante, profesores, En contraposición, un $16.4 \%$ y $19.4 \%$ profesoras, empleados manifestó que los profesores realizan administrativos o de intendencia, estas conductas frecuentemente y muy empleadas administrativas o de frecuentemente, respectivamente.

intendencia, y nadie. Lo manifestado

Ahora bien, en el tercer por la muestra se captura en las apartado del cuestionario (ilustraciones siguientes gráficas:

\section{Ilustración 3}

Piropos o comentarios no deseados acerca de tu apariencia. 
Periódico do Núcleo de Estudos e Pesquisas sobre Gênero e Direito Centro de Ciências Jurídicas - Universidade Federal da Paraíba V. 9 - $\mathrm{N}^{\mathrm{o}} 01$ - Ano 2020 - Spanish Edition ISSN | 2179-7137 | http://periodicos.ufpb.br/ojs2/index.php/ged/index

100

75

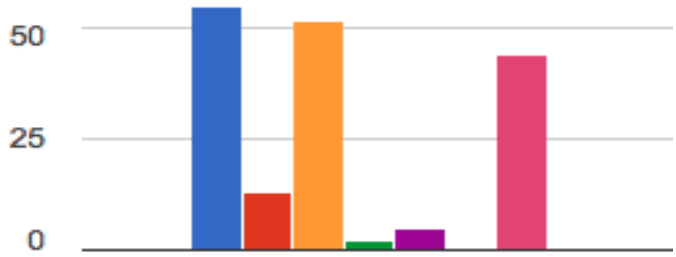

Ilustración 4

Bromas o comentarios inapropiados de índole sexual que te hacen sentir incómoda.

100

75

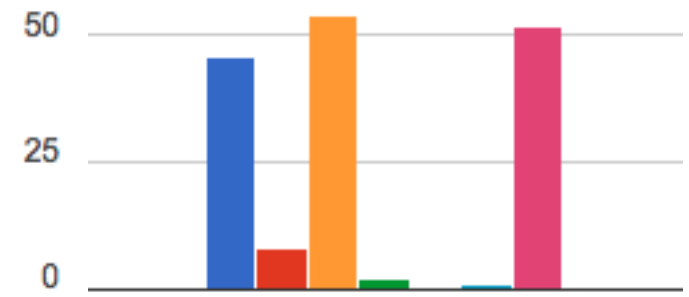

Ilustración 5

Miradas lascivas o gestos morbosos que te incomoden.

100

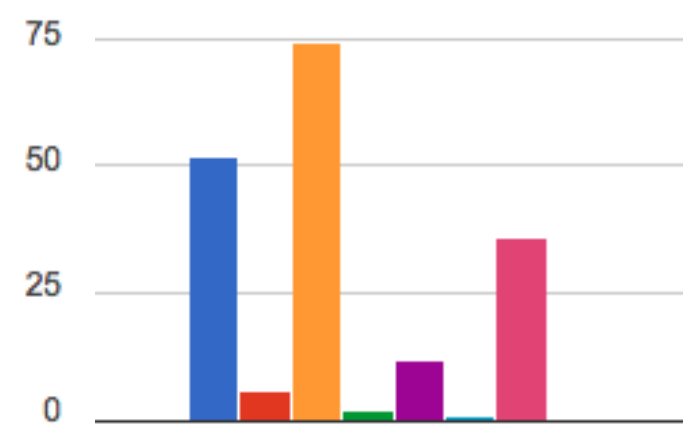


Periódico do Núcleo de Estudos e Pesquisas sobre Gênero e Direito

Centro de Ciências Jurídicas - Universidade Federal da Paraíba

V. 9 - $\mathrm{N}^{\mathrm{o}} 01$ - Ano 2020 - Spanish Edition

ISSN | 2179-7137 | http://periodicos.ufpb.br/ojs2/index.php/ged/index

198

Ilustración 6

Burlas bromas, comentarios o preguntas acerca de tu vida sexual que te incomoden.

100

75

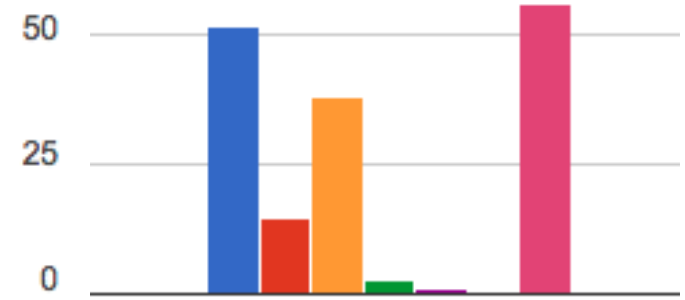

Ilustración 7

Mensajes y/o llamadas haciéndote invitaciones o insinuaciones inapropiadas por celular, redes sociales o alguna otra forma de comunicación digital.

100

75

50

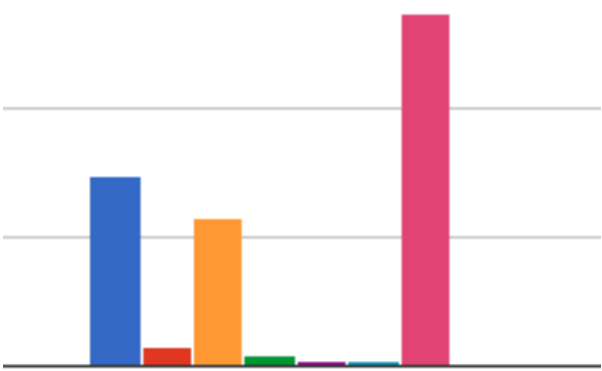

Ilustración 8

Cartas y/o mensajes haciéndote invitaciones y/o insinuaciones inapropiadas por vías de comunicación no digital. 
100

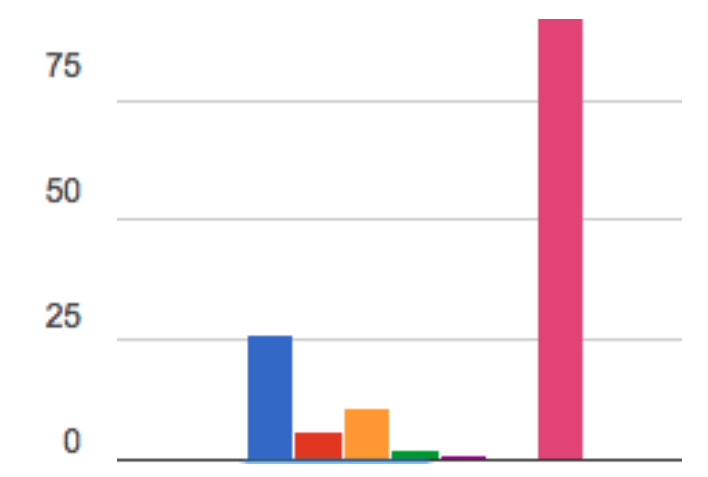

Ilustración 9

Roses/abrazos/besos/tocamientos o alguna forma de violencia física y/o sexual. 100

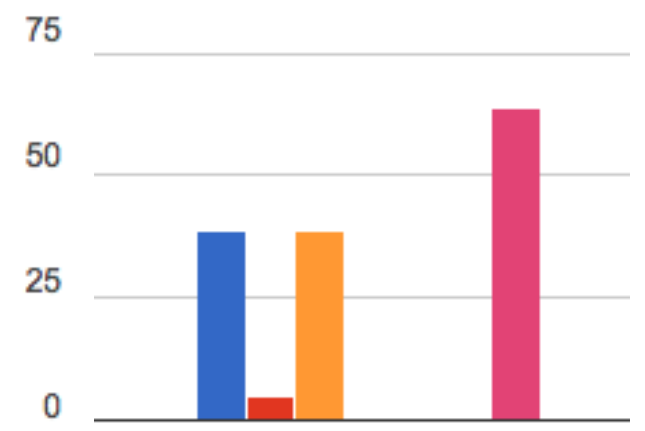

Los datos presentados en las ilustraciones anteriores son alarmantes, pues indican un alto índice de profesores y compañeros realizando conductas que configuran acoso sexual hacia las estudiantes del campus universitario. Los resultados obtenidos sugieren una grave y reiterada ocurrencia de conductas que menoscaban la seguridad, confianza y bienestar de las estudiantes.

En la cuarta sección de la encuesta se realizaron "preguntas respecto a la vida cotidiana en la Facultad". Así, en el numeral 18 del cuestionario se preguntó a las estudiantes cuan a menudo se enteran de que alguna compañera sufre acoso sexual de algún tipo proveniente de un profesor, a lo que contestaron que frecuentemente un $41.8 \%$ y $14.9 \%$ muy frecuentemente. De igual forma, cuando se les preguntó lo mismo respecto a las profesoras (numeral $19 \mathrm{del}$ anexo 1), los porcentajes disminuyeron a $3.7 \%$ frecuentemente $y$ 
$0.7 \%$ muy frecuentemente, lo que sugiere que son conductas de conocimiento popular dentro de la FCS.

Por otro lado, se preguntó a las estudiantes sobre la manera de vestir que adoptan para asistir a la universidad, respecto a si deciden a conciencia su vestimenta para evitar acoso sexual de parte de profesores/as, compañeros/as o trabajador/a administrativo/a, dentro de su centro de estudios (preguntas 20, 21 y 22, respectivamente). Los resultados obtenidos fueron los siguientes:

Tabla 3

\begin{tabular}{|c|c|c|c|}
\hline & Frecuentemente & $\begin{array}{c}\text { Muy } \\
\text { frecuentemente }\end{array}$ & Siempre \\
\hline Profesores/as & $15.7 \%$ & $14.2 \%$ & $24.6 \%$ \\
\hline Compañeros/as & $9.7 \%$ & $14.9 \%$ & $13.4 \%$ \\
\hline $\begin{array}{l}\text { Trabajador/a } \\
\text { administrativo/a o de } \\
\text { intendencia. }\end{array}$ & $11.2 \%$ & $4.5 \%$ & \\
\hline
\end{tabular}

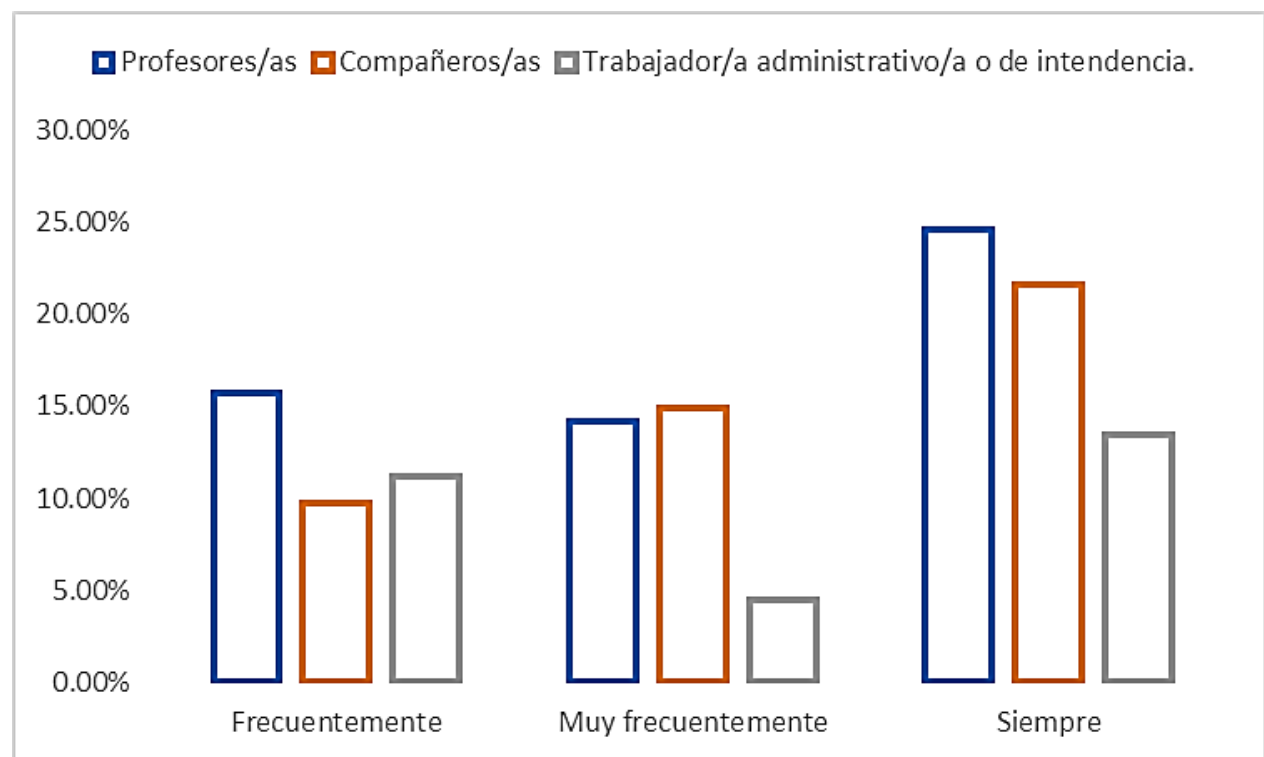

Es de destacarse la pregunta 23 del cuestionario, que indaga si las alumnas al elegir su carga de asignaturas evitan tomar clase con algunos profesores para así también evitar ser víctima de acoso sexual por 
parte de los mismo. En este rubro, un preocupante $44.8 \%$ de la muestra manifestó que siempre que puede evita a determinados profesores, mientras que el $28.4 \%$ manifestó que a veces.

Seguidamente, las alumnas coincidieron en un $55.2 \%$ muy de acuerdo y $35.2 \%$ de acuerdo en que los casos de acoso sexual más habituales dentro de la FCS son perpetrados por profesores; mientras que el $14.9 \%$ muy de acuerdo y $41.8 \%$ de acuerdo, en que los casos de acoso sexual más habituales son perpetrados por estudiantes. Sólo el $4.5 \%$ manifestó estar muy de acuerdo y $14.2 \%$ de acuerdo con que el acoso es perpetrado por personal administrativo y de intendencia.

Por último, en la quinta sección del cuestionario, titulada "Percepción del tratamiento Institucional del acoso y la violencia sexual", las alumnas perciben una falta de actuación, reconocimiento y tratamiento del acoso sexual dentro de la FCS de la USM, según indican los siguientes datos:

- $\quad$ Se les preguntó (27) si consideraban que existieran normas, criterios y protocolos de actuación suficientemente claros para responder en casos de acoso y violencia sexual. La respuesta que dominó la encuesta fue "no" con un $62.7 \%$.

- $\quad$ Se pidió en la pregunta 28 que indicaran si consideran que está garantizado que quien ejecuta algún acto de acoso y/o violencia sexual será sancionado/a, a lo que el $73.9 \%$ contestó que "no", un $12.7 \%$ señaló que "tal vez" y un $13,4 \%$ que "sí".

- Respecto de si se realizan esfuerzos amplios y suficiente para combatir el acoso y la violencia sexual por parte de las autoridades universitarias (pregunta 29), las alumnas manifestaron que se encontraban $38.1 \%$ muy en desacuerdo y $40.3 \%$ en desacuerdo con esa afirmación.

- En lo referente a si se considera que las autoridades universitarias deben implementar mayores medidas para prevenir $\mathrm{y}$ erradicar el acoso y violencia sexual (30), un $73.1 \%$ de la población estudiada expresó estar muy de acuerdo y $17.9 \%$ de acuerdo.

- $\quad$ Sobre si hay un problema estructural y generalizado de acoso u hostigamiento sexual hacia las alumnas (numeral 31 del cuestionario), los datos obtenidos revelan que la amplia mayoría de las estudiantes así lo perciben, pues 
expresó estar de acuerdo con dicha afirmación un $43.3 \%$ de la muestra y muy de acuerdo un $36.6 \%$.

$$
\text { - La pregunta } 32 \text { pidió a al }
$$
grupo estudiado que manifestara si la dignidad personal e integridad sexual de las alumnas está debidamente salvaguardada por las normas $\mathrm{y}$ autoridades institucionales, sobre lo que el $43.3 \%$ señaló estar en desacuerdo y $26.1 \%$ muy en desacuerdo.

- $\quad$ Para concluir, la encuesta indagó en el numeral 33 el grado de acuerdo de la población con la afirmación: "La Facultad de Ciencias Sociales es un espacio donde me siento libre y segura de no sufrir alguna forma de acoso, hostigamiento o violencia sexual", respecto de la cual el $19.4 \%$ de las alumnas manifestaron encontrarse muy en desacuerdo y $49.3 \%$ en desacuerdo.

Así, tras la constatación de los resultados expuestos a lo largo de esta sección, se observa que, en la percepción del grupo social de interés, existe un problema estructural de acoso sexual sufrido por las estudiantes de la FCS de la USM, y que el complejo de conductas involucrados en tal problema estructural es principalmente ejercido por hombres
- prominentemente por profesores - y que se percibe una importante falta de involucramiento de la universidad en el combate al problema. Luego, los datos obtenidos sustentan lo siguiente:

a) Las estudiantes no se sienten seguras en el ambiente escolar, toman medidas especiales en su rutina diaria para evitar ser víctimas de acoso sexual y están conscientes en su mayoría de la existencia de dichas conductas a su alrededor.

b) Las conductas de acoso sexual no se tratan de casos aislados, si no de conductas generalizadas y sistematizadas dentro de un campus que permite a los hombres que conforman su cuerpo académico, administrativo $\mathrm{y}$ estudiantil realizar conductas inapropiadas impunemente, sin tener medidas de seguridad necesarias para asegurarse de no poner en riesgo a sus estudiantes.

c) Las estudiantes consideran una conducta omisiva de la universidad no protegerlas de los perpetradores en temas de acoso sexual, y están conscientes de que son conductas que no se castigan, sabiendo que no cuentan con protocolos de actuación y/o legislación universitaria lo 
suficientemente robusta y adecuada para su protección.

\section{Discusión y conclusiones}

De acuerdo con los datos que se han presentado, no parece muy arriesgado afirmar que la percepción social imperante entre las estudiantes de la FCS de la USM es que el acoso sexual dirigido a las alumnas dentro del campus educativo es un fenómeno lamentablemente - cotidiano. En lo referente a bromas o comentarios
203

inapropiados de índole sexual y miradas lascivas o gestos morbosos, los datos indican que los profesores son los principales perpetradores. Sin embargo, en lo referente a piropos o comentarios no deseados sobre la apariencia, los estudiantes lo son. Cabe destacarse también que los roses, abrazos, besos o violencia física y sexual, son comportamientos cuya autoría es atribuida en igual proporción a profesores y estudiantes (ver tabla 4).

Tabla 4

\begin{tabular}{|c|c|c|c|}
\hline Conducta \% & Profesores & Compañeros & $\begin{array}{l}\text { Personal } \\
\text { administrativo o de } \\
\text { intendencia }\end{array}$ \\
\hline 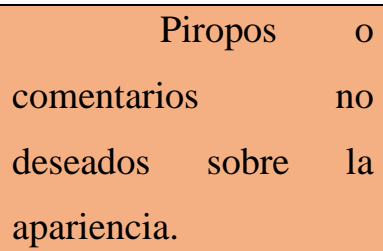 & 41.0 & 38.8 & 3.7 \\
\hline $\begin{array}{l}\text { Bromas o } \\
\text { comentarios } \\
\text { inapropiados de índole } \\
\text { sexual. }\end{array}$ & 34.2 & 40.2 & 0 \\
\hline \begin{tabular}{l}
\multicolumn{3}{c}{ Miradas } \\
lascivas o gestos \\
morbosos.
\end{tabular} & 38.8 & 55.2 & 8.9 \\
\hline $\begin{array}{ll} & \text { Burlas, } \\
\text { bromas } & \text { o preguntas }\end{array}$ & 38.8 & 28.3 & .74 \\
\hline
\end{tabular}


Periódico do Núcleo de Estudos e Pesquisas sobre Gênero e Direito Centro de Ciências Jurídicas - Universidade Federal da Paraíba V. 9 - $\mathrm{N}^{\mathrm{o}} 01$ - Ano 2020 - Spanish Edition ISSN | 2179-7137 | http://periodicos.ufpb.br/ojs2/index.php/ged/index

\begin{tabular}{|c|c|c|c|}
\hline $\begin{array}{ll}\text { incomodas sobre su } \\
\text { vida sexual. }\end{array}$ & & & \\
\hline $\begin{array}{l}\text { Mensajes у о } \\
\text { llamadas, por vías } \\
\text { electrónicas. }\end{array}$ & 27.6 & 20.89 & .74 \\
\hline \begin{tabular}{lr}
\multicolumn{1}{c}{ Cartas } & y \\
mensajes & con \\
invitaciones & o \\
insinuaciones & por \\
medios no electrónicos.
\end{tabular} & 19.4 & 8.2 & .74 \\
\hline $\begin{array}{l}\text { Roces, } \\
\text { abrazos, besos o algún } \\
\text { tipo de violencia física } \\
\text { o sexual }\end{array}$ & 29.1 & 29.1 & 0 \\
\hline
\end{tabular}

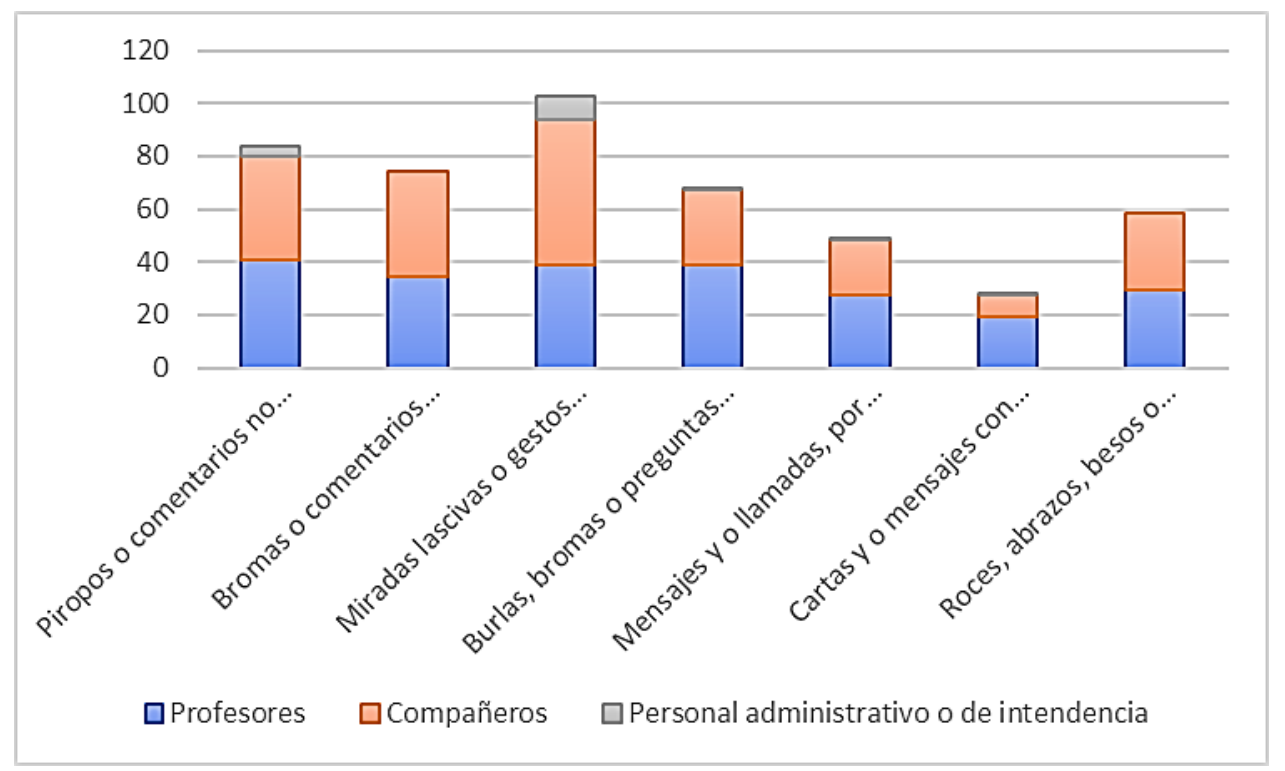

Los siguientes datos (tabla 5) revelan que un porcentaje de mujeres estudiantes - que en la mayoría de los casos rebasa la mitad de la muestra señala que los profesores realizan comentarios que estereotipan o denigran mujeres en mayor medida que a los hombres, que realizan bromas de índole sexual en el aula, preguntan a las alumnas sobre su vida sexual, hacen comentarios sobre su apariencia, provocan que las alumnas cambien su 
modo de vestir o consideren el riesgo de sufrir acoso como factor para la elección de asignaturas, así como que el $91 \%$ de las alumnas señala que los más habituales casos de acoso son realizados por profesores, superando el $56.7 \%$ que señalo que eran los compañeros estudiantes y el $18.5 \%$ que señaló que era el personal administrativo y de intendencia.

\section{Tabla 5}

\begin{tabular}{|c|c|c|c|c|}
\hline $\begin{array}{l}\text { Conduc } \\
\text { ta (Frecuente o } \\
\text { muy } \\
\text { frecuentemente) } \\
\%\end{array}$ & Profe & $\begin{array}{ll} & \text { Profe } \\
\text { soras } & \end{array}$ & $\begin{array}{ll}\text { Compa } & \text { neros }\end{array}$ & $\begin{array}{l}\text { Pers } \\
\text { onal } \\
\text { administrativ } \\
0 \quad \text { de } \\
\text { intendencia }\end{array}$ \\
\hline $\begin{array}{l}\text { Bromas } \\
\text { que estereotipan o } \\
\text { denigran a las } \\
\text { mujeres. }\end{array}$ & 51.5 & 20.9 & - & - \\
\hline $\begin{array}{l}\text { Bromas } \\
\text { o comentarios } \\
\text { inapropiados de } \\
\text { índole sexual. }\end{array}$ & 55.2 & 13.4 & - & - \\
\hline $\begin{array}{l}\text { Pregunta } \\
\text { s incomodas sobre } \\
\text { la vida sexual de } \\
\text { las alumnas. }\end{array}$ & 38.1 & 8.2 & - & - \\
\hline $\begin{array}{l}\text { Piropos } \\
\text { o comentarios no } \\
\text { deseados sobre la } \\
\text { apariencia de las } \\
\text { alumnas. }\end{array}$ & 59 & 10.5 & - & - \\
\hline $\begin{array}{l}\text { Conoci } \\
\text { miento de una } \\
\text { compañera }\end{array}$ & 56.7 & 4.4 & - & - \\
\hline
\end{tabular}


Periódico do Núcleo de Estudos e Pesquisas sobre Gênero e Direito Centro de Ciências Jurídicas - Universidade Federal da Paraíba V. 9 - $\mathrm{N}^{\mathrm{o}} 01$ - Ano 2020 - Spanish Edition ISSN | 2179-7137 | http://periodicos.ufpb.br/ojs2/index.php/ged/index

\begin{tabular}{|c|c|c|c|c|}
\hline $\begin{array}{l}\text { sufriendo acoso } \\
\text { sexual }\end{array}$ & & & & \\
\hline $\begin{array}{l}\text { Elegir } \\
\text { como irán vestidas } \\
\text { para no sufrir } \\
\text { acoso sexual. }\end{array}$ & \multicolumn{2}{|l|}{54.5} & 46.2 & 29.1 \\
\hline \begin{tabular}{lrr} 
& \multicolumn{2}{c}{ Evitar } \\
profesores & por \\
temor & a & ser \\
víctima & de & acoso \\
sexual. & &
\end{tabular} & 73.2 & - & - & - \\
\hline
\end{tabular}

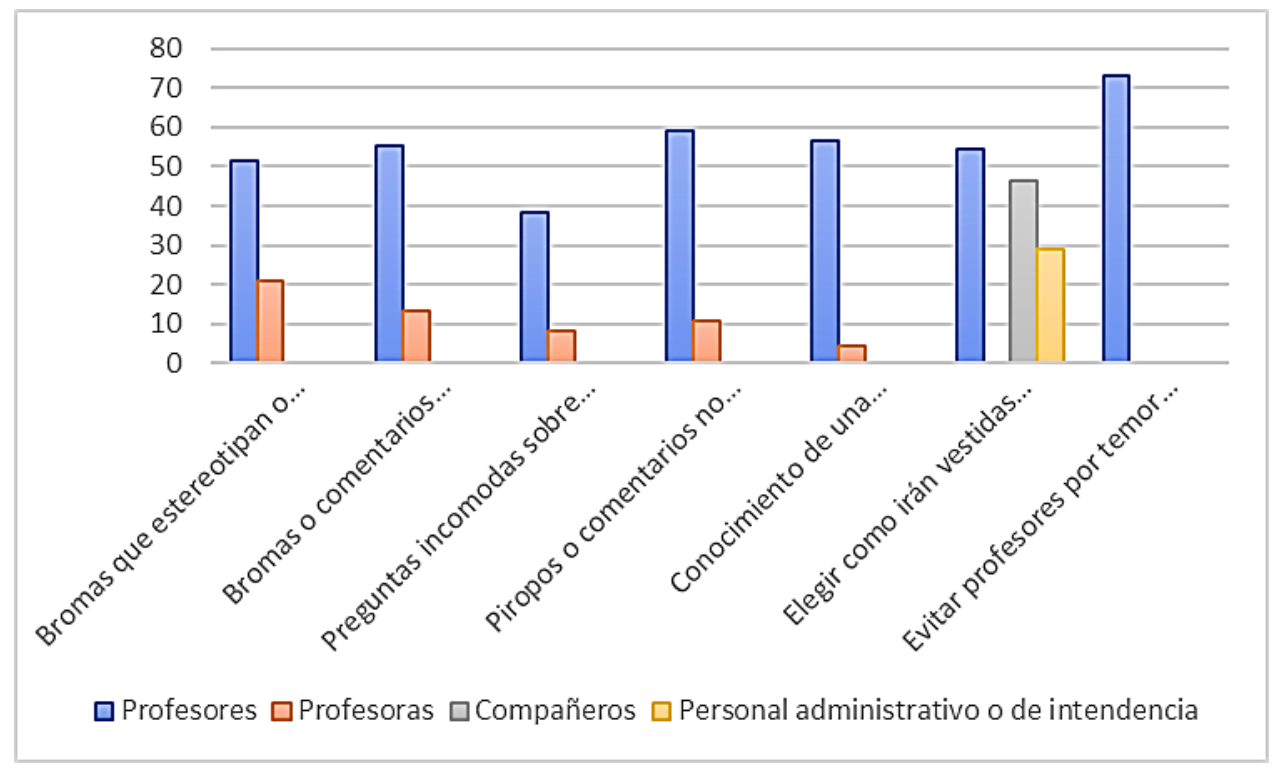

Finalmente, el rubro del de respuesta claros; b) no están cuestionario referente al abordaje garantizadas las sanciones para los institucional del acoso sexual en el perpetradores; c) las autoridades espacio universitario entrega resultados institucionales no realizan los suficientes dignos de atención. Según los datos esfuerzos para lidiar con y prevenir el recopilados, la percepción social acoso sexual en el espacio universitario imperante entre la estudiantes es que a) y para salvaguardar la dignidad personal no existen normas, criterios ni protocolos e integridad sexual de las alumnas; y d) 
que la mayoría de las alumnas no consideran a la FCS de la USM un espacio seguro y libre de acoso sexual.

Por todo lo anterior, podemos afirmar que los datos recolectados nos permiten dar por cumplidos los objetivos pues ahora conocemos:

a) Que, en la percepción social de la población estudiada, en efecto hay un problema sistemático de acoso sexual sobre las estudiantes de la FCS de la USM y que sus principales autores son los profesores;

b) Cuál es la percepción que la población estudiada tiene respecto de las conductas consideradas como formas de acoso sexual y la frecuencia con la que estiman que son perpetradas; $\mathrm{y}$

c) Que el grado de disconformidad que la población estudiada manifiesta respecto de la respuesta y tratamiento institucional de la situación es alto.

\section{Recomendaciones}

\section{prospectiva}

Los datos expuestos en el apartado de resultados son alarmantes e invitan a plantear medidas y políticas de intervención social contundentes que en el futuro logren prevenir, identificar, denunciar y erradicar la conducta de acoso sexual dentro del espacio educativo.

Pueden considerarse como primera y más obvia medida de prevención la concientización y sensibilización tanto para la víctima como para el perpetrador de la conducta. Los datos obtenidos exhiben la necesidad de establecer campañas informativas que cataloguen y enseñen a reconocer los comportamientos inadecuados que se han identificado en el estudio, con la intención de desalentar su realización o intuir su constitución, con la expectativa de que la comunidad estudiantil sea competente para combatir las conductas que ponen en riesgo su integridad y la de otras estudiantes.

La prevención e identificación de la conducta es una fase necesarísima dentro de la estrategia de erradicación del acoso sexual en el espacio educativo. Sin embargo, se requiere la estructuración institucional de medidas que amonesten la ejecución de los comportamientos descritos páginas atrás.

En ese orden de ideas, es digna de considerarse la posibilidad de establecer un sistema de vigilancia o la 
creación de un observatorio de violencia contra la mujer que cuente con autonomía para la toma de decisiones y la implementación de políticas y medidas de acción que garanticen la protección de las estudiantes. Dicho observatorio deberá, entre otras cosas, medir la incidencia del acoso y la violencia sexual sufrida por las estudiantes universitarias y, en línea de principio, debería estar integrado de manera bilateral, es decir, deberá contar tanto con representantes institucionales como con representantes estudiantiles. Se trata así mismo de un cuerpo que deberá tener la capacidad jurídica de recibir y dar debido seguimiento a las denuncias estudiantiles, diseñar procesos para calcular los índices de acoso existentes, catalogar las conductas en razón de su gravedad, llevar un registro de responsables y emitir sanciones o recomendaciones de sanción para los sujetos responsables de las conductas lesivas.

\section{Referencias bibliográficas}

Asamblea General de la Organización de Estados Americanos (9 de junio de 1994). Convención Interamericana para prevenir, sancionar y erradicar la
208

violencia contra la mujer "Convención Belem do Pará". DO: 19 de enero de 1999. Recuperado de: http://www.ordenjuridico.gob.mx/TratI nt/Derechos\%20Humanos/D1BIS.pdf (22 de noviembre de 1969). Convención Americana sobre Derechos Humanos. DO: 7 de mayo de 1981. Recuperado de: http://www.ordenjuridico.gob.mx/TratI nt/Derechos\%20Humanos/D1BIS.pdf

Asamblea General de la Organización de las Naciones Unidad (10 de diciembre de 1948). Declaración Universal de los Derechos Humanos. [Resolución 217 A (III)]. Recuperado de: https://www.ohchr.org/EN/UDHR/Docu ments/UDHR_Translations/spn.pdf

Estados Unidos Mexicanos. (1 de febrero de 2007). Ley General de Acceso a las Mujeres a una Vida Libre de Violencia. DO: 1 de febrero de 2007. Recuperado de:

http://www.diputados.gob.mx/LeyesBib lio/pdf/LGAMVLV_130418.pdf

Hoagland, S. (2000).

"Heteropatriarchy". En Encyclopedia of 
Periódico do Núcleo de Estudos e Pesquisas sobre Gênero e Direito Centro de Ciências Jurídicas - Universidade Federal da Paraíba V. 9 - $\mathrm{N}^{\mathrm{o}} 01$ - Ano 2020 - Spanish Edition ISSN | 2179-7137 | http://periodicos.ufpb.br/ojs2/index.php/ged/index

Feminist Theories de Code, L. (Ed.).

New York: Routledge, p. 245 